\title{
SATURNIAN LOW FREQUENCY DRIFTING RADIO BURSTS: STATISTICAL PROPERTIES AND POLARIZATION
}

\author{
U. Taubenschuss*, J. S. Leisner*, G. Fischer ${ }^{\dagger}$, \\ D. A. Gurnett*, and F. Nemec*
}

\begin{abstract}
After Cassini's arrival at planet Saturn, its Radio and Plasma Wave Science (RPWS) experiment has performed numerous observations of a new type of planetary radio emissions in the lower $\mathrm{kHz}$ frequency range $(<50 \mathrm{kHz})$. These bursty emissions have time scales of a few to 15 minutes and occur as slowly drifting events in the time-frequency spectrogram. They have neither been detected by the Voyager spacecraft nor by Ulysses. As a first approach to this new phenomenon, results of a statistical study with regard to the observer's position, i.e. Cassini's orbital position, will be presented. Furthermore, aspects of polarization will be highlighted as far as appropriate goniopolarimetric (3-antenna) observations are available.
\end{abstract}

\section{Introduction}

The Cassini spacecraft has been orbiting the gas giant Saturn since July 2004. Its RPWS instrument is designed to measure plasma wave phenomena as well as electromagnetic waves which are generated by complex interactions between the planetary magnetic field and the magnetospheric/ionospheric plasma environment. This experiment includes three nearly orthogonal monopole antennas $(u, v, w)$, which are sensitive to oscillations of the electric field component. These monopoles can be connected to a system of five different receivers, enabling a nearly continuous survey of waves inside a frequency range of 1 $\mathrm{Hz}$ up to $16 \mathrm{MHz}$ [Gurnett et al., 2004]. For this study we use data from bands A $(3.5-16 \mathrm{kHz})$ and $\mathrm{B}(16-71 \mathrm{kHz})$ of the High Frequency Receiver (HFR). Most of the time, the monopoles $u$ and $v$ are combined to a dipole antenna providing a standard survey mode with increased spectral resolution. On the other hand, the HFR can be operated in a 3-antenna or goniopolarimetric mode. In this mode, the signals from all three monopoles are processed thus yielding enough independent measurements for a

\footnotetext{
* Department of Physics and Astronomy, University of Iowa, Iowa City, IA 52242, USA

$\dagger$ Space Research Institute, Austrian Academy of Sciences, Schmiedlstrasse 6, A-8042 Graz, Austria
} 
complete polarization analysis. One gets the four Stokes parameters $S, Q, U, V$ and two direction angles defining the direction towards the radio source in the spacecraft frame [Cecconi and Zarka, 2005].

On July 2 (DOY 184), 2004, directly after the Saturn orbit insertion maneuver, the HFR detected faint emissions at $5-25 \mathrm{kHz}$, which exhibit a burst-like pattern, a quasiperiodic repetition, and a characteristic negative drift when displayed in a time-frequency spectrogram. We introduce this new type of Saturnian radio emission by outlining its spectral characteristics (see Section 2). The main part of this work focusses on a statistical analysis on the basis of more than five years of data recorded by Cassini (mid-2004 to the end of 2009). Section 3 highlights the dependence of visibility of these drifting bursts with regard to the observer's orbital position around Saturn. Section 4 discusses the polarization using data which are obtained in the 3-antenna mode. Finally, conclusions are drawn in Section 5.

\section{Spectral Characteristics}

At this point, the acronym SDBs is introduced for the drifting radio events, meaning Saturn Drifting Bursts. SDBs are detected by the RPWS/HFR, typically at frequencies below $50 \mathrm{kHz}$. Their lower frequency limit sometimes extends below the lower cutoff frequency of the HFR $(3.5 \mathrm{kHz})$ and remains, so far, unknown until data archives from Cassini's Medium Frequency Receiver (MFR) or the Wideband Receiver (WBR) have been evaluated. A good example of SDBs, which has been recorded on DOY 44, 2007, is presented in Fig. 1. Emissions identified as SDBs are marked with the labels "down" and "up". Faint upward drifting structures are present around $30 \mathrm{kHz}$. Two downward drifting bands of emission can be identified around $10 \mathrm{kHz}$ and $20 \mathrm{kHz}$. The first hour of observation is in the 3-antenna mode which has a temporal resolution of 32 seconds and 13 logarithmically spaced frequency channels inside the displayed range of $3.6-$ $40 \mathrm{kHz}(\Delta f / f=20 \%)$. It demonstrates that the spectral features of SDBs can hardly be determined at such low spectral resolutions. Spectral fine structures are better visible in 2-antenna data with a temporal resolution of 16 seconds and 52 frequency channels covering the displayed range $(\Delta f / f=5 \%)$.

A closer look at Fig. 1 reveals that frequencies of the upper band around $20 \mathrm{kHz}$ are exactly twice those of the lower band around $10 \mathrm{kHz}$. This simultaneous visibility of SDBs at the fundamental frequency and its first harmonic occurs in about $1 / 3$ of observed cases. An artificial generation of the harmonics due to a distortion effect in the receiver can be ruled out because higher order harmonics are absent. Furthermore, the fundamental and the harmonic have opposite signs of circular polarization as can be inferred from cross correlation spectra obtained in the 3-antenna mode. If both emissions are generated at approximately the same source region, then opposite polarizations imply different wave modes. One corresponds to the LO-mode and the other one to the RX-mode. This is comparable to the $20 \mathrm{kHz}$ narrowband emission or to Saturn kilometric radiation (SKR), which are also generated either as O-mode or X/Z-mode emission [Ye et al., 2009; Lamy et al., 2008]. Two bands of narrowband emission are marked in Fig. 1 at $\sim 13 \mathrm{kHz}$ and $\sim 26 \mathrm{kHz}$ (see labels "NB"). It is often observed that SDBs and narrowband emissions 


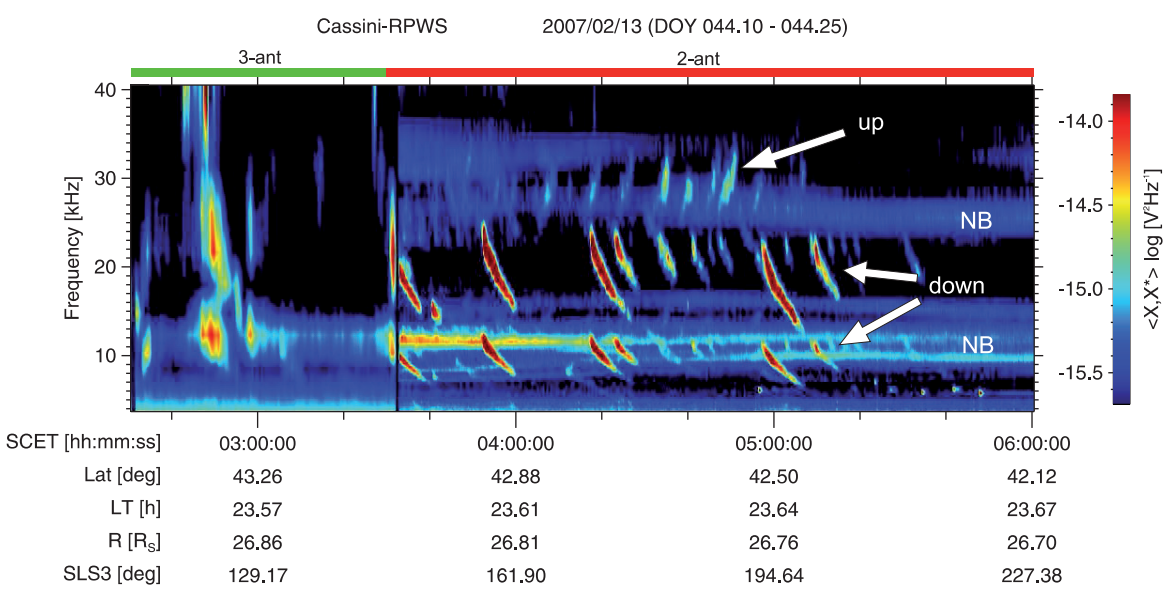

Figure 1: Cassini RPWS dynamic spectrum recorded on DOY 44, 2007, containing SDB emissions with negative ("down") and positive ("up") drift rates. Narrowband emissions ("NB") are also marked. $\left\langle X, X^{*}\right\rangle$ stands for the auto-correlation signal in the u-antenna during 3-antenna measurements and for the auto-correlation in the dipole antenna during 2-antenna measurements.

overlap in dynamic spectra or smoothly morph into each other. Nevertheless, both types of emission can be clearly distinguished according to their different spectral characteristics. Repetitive drifting structures on time scales of several minutes are neither linked to typical patterns of narrowband emission nor to SKR. Narrowband emission is a product of mode conversion [Menietti et al., 2010], so a similar generation mechanism might be suggested for SDBs. Mode conversion does not exclude a possible generation of SDBs in the Zmode. However, SDBs are observed at frequencies clearly above the local upper hybrid frequency, which corresponds to the resonance frequency for the Z-mode. Thus, only the two modes $\mathrm{O}$ and $\mathrm{X}$ are allowed to propagate.

One single SDB, i.e. one radio burst, typically lasts for $1-15$ minutes. During this time, its frequency is changing steadily by about $1-10 \mathrm{kHz}$. A polynomial fit to SDBs' time-frequency contours of the form

$$
f=D_{0}+D_{L} *\left(t-t_{0}\right)+D_{Q} *\left(t-t_{0}\right)^{2}
$$

( $f \ldots$ frequency, $t \ldots$ time, $t_{0} \ldots$ start time of SDB) yields linear drift rates $\left(D_{L}\right)$ of $0.3-3$ $\mathrm{kHz} / \mathrm{min}$. In $85 \%$ of observed cases, linear drift rates are negative, i.e. the frequency is decreasing with increasing time (marked with "down" in Fig. 1). Nevertheless, also positive linear drift rates can be observed, typically at higher frequencies above $15 \mathrm{kHz}$ (marked with "up" in Fig. 1). In addition to the dominant linear drift, a small quadratic drift term $\left(D_{Q}\right)$ introduces a slight curvature. Both the linear $\left(D_{L}\right)$ and the quadratic $\left(D_{Q}\right)$ drift coefficients depend on frequency. For negative drifts, $D_{L}$ can be fitted by $D_{L} \approx-f_{d c} / 13\left(f_{d c}\right.$ in $\mathrm{kHz}, D_{L}$ in $\left.\mathrm{kHz} / \mathrm{min}\right)$. Here, $f_{d c}$ corresponds to the SDB's center frequency. For positive drifts, $D_{L} \approx f_{d c} / 11$. Any dependence of $D_{Q}$ with $f_{d c}$ can not 
be determined with sufficient accuracy due to the HFR's limited frequency resolution, especially at higher frequencies.

\section{Visibility Depending on Cassini's Position}

Correlations between SDB observations and all basic position parameters of Cassini are summarized in Fig. 2, showing the dependence on (a) radial distance, (b) kronographic latitude, (c) SLS4 longitude and (d) local time. These histograms reflect the amount of time during which SDBs are visible by counting the number of 5-minute intervals which compose a period of continuous observation. Additionally, histograms are normalized by the amount of time Cassini spent in a certain position bin in order to compensate for the very special orbit geometry during the observations. As can be seen from Fig. 2a, the visibility is very low at distances $r<8 \mathrm{R}_{\mathrm{S}}$, followed by a broad peak between $8-20 \mathrm{R}_{\mathrm{S}}$ and two sharper peaks at $r \sim 30 \mathrm{R}_{\mathrm{S}}$ and at $r \sim 45 \mathrm{R}_{\mathrm{S}}$. Peaks at greater radial distances can be considered as statistically insignificant due to the small amount of data Cassini has recorded there. Neglecting the sharper peaks, there is a more or less gradual decrease between $15-50 R_{S}$. This might be a direct consequence of the inverse square fall-off of radio intensities with distance, if the source regions are within $8-15 \mathrm{R}_{\mathrm{S}}$. The two sharper peaks at $r \sim 30 \mathrm{R}_{\mathrm{S}}$ and at $r \sim 45 \mathrm{R}_{\mathrm{S}}$ might reflect the fact that at larger distances to Saturn multiple sources for SDBs come into view thus extending the periods of visibility if Cassini is in a proper position along its orbit, e.g. with regard to latitude. Fig. 2a also includes some observations at larger radial distances, which have been made outside the Saturnian magnetosphere. The most distant observation of SDBs was made on DOY 278,2004 , at $r=110 \mathrm{R}_{\mathrm{S}}$. This confirms that SDBs are electromagnetic emissions which propagate in the free-space modes $\mathrm{O}$ and/or X, because plasma parameters change many orders of magnitude between Saturn's inner magnetosphere and the magnetopause.

The dependence of observations with regard to the observer's kronographic latitude is shown in Fig. $2 \mathrm{~b}$. Two prominent peaks are evident around $\pm 40^{\circ}$ latitude. This suggests a preferential beaming of radiation into the mid-latitude sector.

Fig. 2c presents the occurrence of Cassini's SLS4 longitude during SDB observations. The SLS4 system takes into account the different magnetospheric rotation periods of Saturn's northern and southern hemisphere [Gurnett et al., 2009; Gurnett et al., 2011]. For a clear separation with regard to hemisphere, only observations at $>10^{\circ}$ latitude above and below the equatorial plane contribute to this plot. The normalized occurrences of SLS4-south (light grey) are shifted by -0.2 and over-plotted above SLS4-north (dark grey). SLS4-south exhibits a minimum around $0^{\circ}$ and rather random variations between $0^{\circ}-360^{\circ}$, which might be the result of scarce southern hemisphere observations $(\sim 20 \%$ of all data). On the other hand, northern hemisphere observations ( $\sim 53 \%$ of all data) exhibit a shallow sinusoidal modulation, with a minimum around $90^{\circ}$ and a maximum around $270^{\circ}$.

The visibility with regard to local time (LT) shows strong modulations (Fig. 2d). SDBs are preferentially observed in the pre-noon sector around LT 10:00 and in the midnight sector around LT 24:00. These peaks in the histogram are separated by gaps at LT 06:00 and $\sim 14$ :00. Despite our correction for the time Cassini spends in each bin, the modulation 

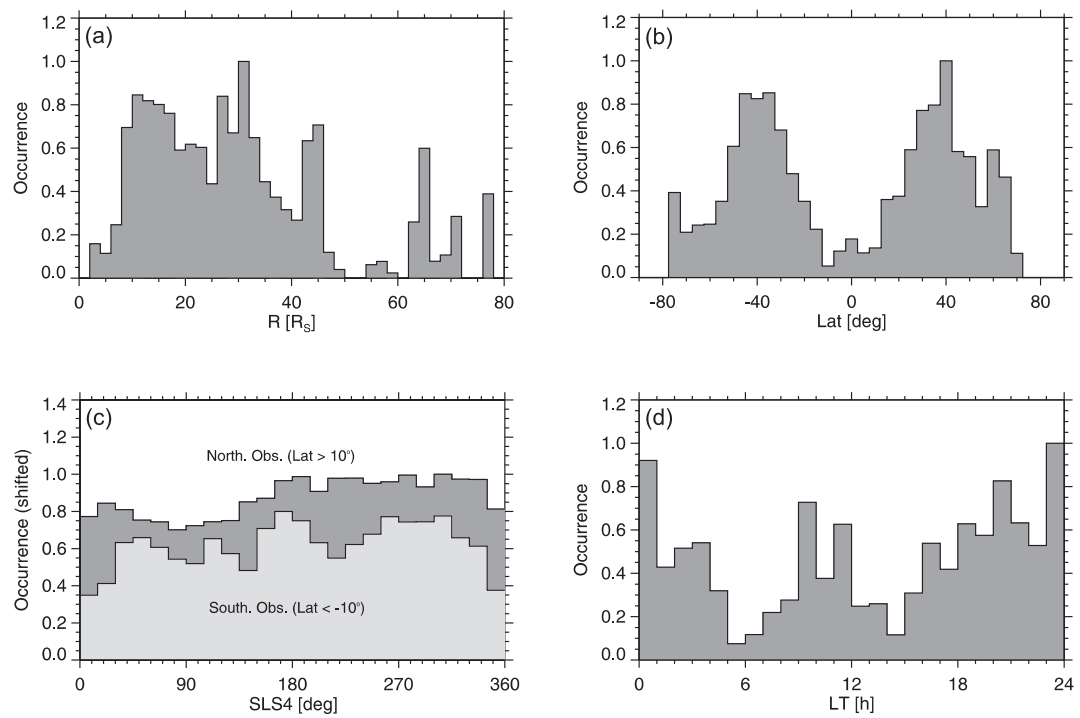

Figure 2: The normalized occurrence rate of SDBs displayed as a function of (a) Cassini's radial distance from Saturn, (b) its kronographic latitude, (c) its SLS4 longitude and (d) its local time.

in local time has to be interpreted with caution. It is strongly correlated with latitude and radial distance due to the geometry of Cassini's orbits during 2004 - 2010. Since the pre-2010 orbits cross the northern hemisphere around dusk and the southern hemisphere around dawn, we have incomplete LT-latitude coverage. Furthermore, crossings of the southern hemisphere below $-20^{\circ}$ latitude are all inside a radial distance of $r<20 \mathrm{R}_{\mathrm{S}}$.

\section{Polarization}

The polarization of SDBs is investigated using 3-antenna mode data in combination with an analytical solution based on a direct inversion [Cecconi and Zarka, 2005]. From the middle of 2004 to the end of 2009 , the 3 -antenna mode has been utilized during $\sim 10 \%$ of the time, yielding 1376 drifting bursts for which the Stokes parameters are determined. The SDBs are digitally scanned in the time-frequency spectrogram and only scanned spectral points belonging to SDBs are considered for a statistical analysis. The results are summarized in Fig. 3, which contains histograms for the occurrence probabilities of (a) spectral flux density or total intensity $S$ as well as histograms for a combination of the Stokes parameters into the degrees of (b) total polarization $d=\left(Q^{2}+U^{2}+V^{2}\right)^{1 / 2} / S$, (c) circular polarization $d_{c}=V / S$, and (d) linear polarization $d_{l}=\left(Q^{2}+U^{2}\right)^{1 / 2} / S$. All scanned data compose the dark grey histograms. Histograms in light grey represent data with a signal-to-noise ratio SNR $>10^{2}$. This reduced data set excludes weak signals close to the background noise level and thus better reflects the true polarization of SDBs. 

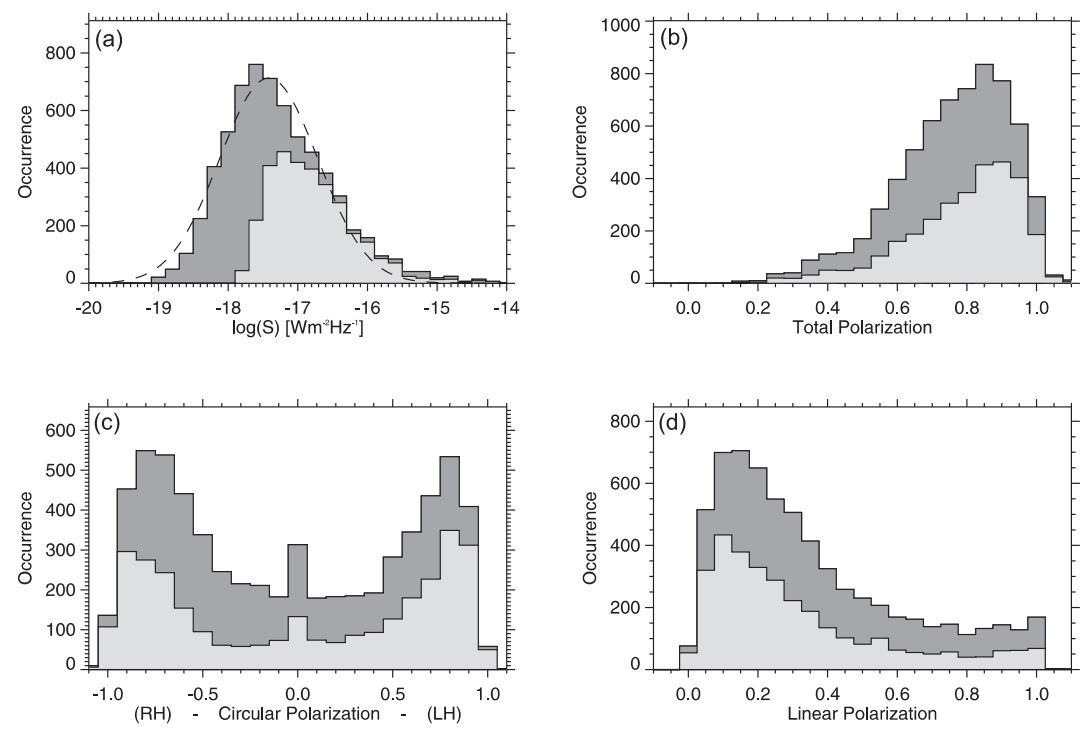

Figure 3: The histograms of (a) total intensity $S$, (b) the degree of total polarization, (c) the degree of circular polarization and (d) the degree of linear polarization. All scanned data are shown in dark grey. Light grey histograms are composed of data points with an SNR $>10^{2}$.

The histogram of total intensities almost follows a Gaussian distribution function (see Gaussian fit drawn as dashed line in Fig. 3a). The other three histograms reveal that SDBs are highly polarized emissions. The degree of total polarization exhibits a peak around $d=0.90 \pm 0.05$ (see light grey histogram in Fig. 3b) and contains no completely unpolarized values close to $d=0$. The high degree of polarization is mainly due to a high degree of circular polarization as can be concluded from Fig. 3c. Two peaks around \pm 0.85 (error \pm 0.1 ) mark the two possible senses, i.e. left-handed and right-handed. A non-vanishing degree of linear polarization with a peak at $d_{l}=0.10 \pm 0.05$ (see Fig. 3d) finally yields an elliptical polarization for SDBs, although the shape of the polarization ellipse is very close to a circle (ellipticity angle peaks at $\pm 40^{\circ}$; not shown here).

Besides the dominant circular polarization, there is a smaller peak visible around $d_{c} \sim 0$ (Fig. 3c). These values are highly linearly polarized. Their occurrence neither shows a clear correlation with Cassini's position nor a dependence on frequency. The linear component might be real because signal-to-noise ratios clearly exceed the $10^{2}$-threshold [Fischer et al., 2009], but their origin is not yet understood.

As already mentioned in Section 2, the fundamental and its first harmonic have opposite signs of circular polarization. Further, each sign changes with regard to the hemisphere of the observer. In the northern hemisphere, the fundamental is left-handed polarized and the harmonic is right-handed polarized. In the southern hemisphere, the situation reverses to a right-handed fundamental and a left-handed harmonic. Consequently, the fundamental has the same polarization as O-mode SKR (or O-mode NB) while the harmonic has 
that of X-mode SKR.

\section{Summary and Conclusions}

We report on a first statistical analysis of a new type of Saturnian radio emission: the very low frequency drifting radio bursts, or simply called SDBs. These emissions have been detected exclusively by Cassini's RPWS instrument between 3.5 and $50 \mathrm{kHz}$ ( VLF range). Their frequency bandwidth is limited to $10-15 \mathrm{kHz}$. SDBs have never been observed above frequencies of $50 \mathrm{kHz}$, but they sometimes do extend below the lower frequency cutoff of the RPWS High Frequency Receiver. The most characteristic feature is a clear drift in the time-frequency spectrogram. Drift rates, which can be positive or negative, depend on the SDB's center frequency, but fall within $0.3-3 \mathrm{kHz} / \mathrm{min}$. The duration of one drifting burst usually does not exceed 15 minutes, but a complete series of bursts typically lasts for several hours. A repeated sequence of drifting radio bursts is a phenomenon which is observed at various places in our solar system, like the Jupiter decameter S-bursts [Zarka et al., 1997], the striated auroral kilometric bursts at Earth [Mutel et al., 2006], or the solar S-bursts [Melnik et al., 2010]. Drift rates vary over a broad range depending on the plasma and magnetic field configuration at the source region, but burst durations usually fall into the milliseconds or seconds time range. These emissions are closely related to particle beams (electrons or ions) or to propagating disturbances which may destabilize the local plasma distribution function. An analog mechanism is supposed for the Saturnian drifting bursts, but detailed studies are pending until we are able to accurately define the source regions. However, Saturn drifting bursts represent a new outstanding phenomenon with exceptionally long burst durations up to 15 minutes.

A statistical study of Cassini's position during the times of SDB observation constrains possible source regions to a radial range of $8-15 R_{S}$. Furthermore, the emission seems to be beamed into the mid-latitude sectors of both hemispheres. For a more specific definition of source regions, the results of direction finding have to be combined with ray tracing on the basis of a model for the generation of SDBs. The detected $\mathrm{O}$ and $\mathrm{X}$ modes can either be generated directly via the cyclotron maser instability (CMI) [Wu and Lee, 1979], or they are products of a linear or a non-linear mode conversion [Oya, 1974; Jones, 1977; Melrose, 1981]. Proper ray tracing requires the knowledge of the magnetic field and a sophisticated model for the distribution of the plasma density inside Saturn's magnetosphere [Giampieri and Dougherty, 2004; Morooka et al., 2009; Persoon et al., 2009; Schippers et al., 2008]. These points are beyond the scope of this work and will be addressed in a future study.

A polarization analysis reveals that SDBs are highly circularly polarized $\left(d_{c}\right.$ peaks at \pm 0.85 ). Furthermore, the fundamental and its first harmonic, at twice the fundamental frequency, can be visible at the same time. With regard to polarization and Cassini's hemisphere of observation, the fundamental behaves like O-mode SKR, and the first harmonic behaves like X-mode SKR. Another indication for the presence of O-mode and $\mathrm{X}$-mode, i.e. modes which are able to propagate at large angles with respect to the direction of the ambient magnetic field, are numerous detections of SDBs at large radial distances even outside Saturn's magnetosphere. 


\section{References}

Cecconi, B., and P. Zarka, Direction finding and antenna calibration through analytical inversion of radio measurements performed using a system of 2 or 3 electric dipole wire antennas on a three-axis stabilized spacecraft, Radio Sci., 40, RS3003, 2005.

Fischer, G., B. Cecconi, L. Lamy, S.-Y. Ye, U. Taubenschuss, W. Macher, P. Zarka, W.S. Kurth, and D. A. Gurnett, Elliptical polarization of Saturn kilometric radiation observed from high latitudes, J. Geophys. Res., 114, A08216, 2009.

Giampieri, G., and M. Dougherty, Modelling of the ring current in Saturn's magnetosphere, Ann. Geophys., 22, 653-659, 2004.

Gurnett, D. A., et al. (29 co-authors), The Cassini Radio and Plasma Wave investigation, Space Sci. Rev., 114, 1, 395-463, 2004.

Gurnett, D. A., A. Lecacheux, W. S. Kurth, A. M. Persoon, J. B. Groene, L. Lamy, P. Zarka, and J. F. Carbary, Discovery of a north-south asymmetry in Saturn's radio rotation period, Geophys. Res. Lett., 36, L16102, 2009.

Gurnett, D. A., J. B. Groene, T. F. Averkamp, W. S. Kurth, S.-Y. Ye, and G. Fischer, An SLS4 longitude system based on a tracking filter analysis of the rotational modulation of Saturn kilometric radiation, in Planetary Radio Emissions VII, edited by H. O. Rucker, W. S. Kurth, P. Louarn, G. Fischer, Austrian Academy of Sciences Press, Vienna, this issue, 51-64, 2011.

Jones, D., Mode coupling of Z-mode waves as a source of auroral kilometric radiation and Jovian decameter radiation, Astron. Astrophys., 55, 245, 1977.

Lamy, L., P. Zarka, B. Cecconi, R. Prangé, W.S. Kurth, and D. A. Gurnett, Saturn kilometric radiation: Average and statistical properties, J. Geophys. Res., 113, A07201, 2008.

Mel'nik, V. N., A. A. Konovalenko, H. O. Rucker, V. V. Dorovskyy, E. P. Abranin, A. Lecacheux, and A.S. Lonskaya, Solar S-bursts at frequencies of 10-30 MHz, Solar Phys., 264, 103-117, 2010.

Melrose, D. B., A theory for the nonthermal radio continua in the terrestrial and Jovian magnetospheres, J. Geophys. Res., 86, 30, 1981.

Menietti, J. D., P. H. Yoon, S.-Y. Ye, B. Cecconi, and A. M. Rymer, Source mechanism of Saturn narrowband emission, Ann. Geophys., 28, 1013-1021, 2010.

Morooka, M. W., R. Modolo, J.-E. Wahlund, M. André, A. I. Eriksson, A. M. Persoon, D. A. Gurnett, W. S. Kurth, A. J. Coates, G. R. Lewis, K. K. Khurana, and M. K. Dougherty, The electron density of Saturn's magnetosphere, Ann. Geophys., 27, 29712991, 2009.

Mutel, R. L., J. D. Menietti, I. W. Christopher, D. A. Gurnett, and J. M. Cook, Striated auroral kilometric radiation emission: A remote tracer of ion solitary structures, $J$. Geophys. Res., 111, A10203, 2006. 
Oya, H., Origin of Jovian decameter wave emissions-Conversion from the electron cyclotron plasma wave to the ordinary mode electromagnetic wave, Planet. Space Sci., 22, 687-708, 1974.

Persoon, A. M., D. A. Gurnett, O. Santolik, W.S. Kurth, J. B. Fadden, J. B. Groene, G. R. Lewis, A. J. Coates, R. J. Wilson, R. L. Tokar, J.-E. Wahlund, and M. Moncuquet, A diffusive equilibrium model for the plasma density in Saturn's magnetosphere, J. Geophys. Res., 114, A04211, 2009.

Schippers, P., M. Blanc, N. André, I. Dandouras, G. R. Lewis, L. K. Gilbert, A. M. Persoon, N. Krupp, D. A. Gurnett, A.J. Coates, S. M. Krimigis, D. T. Young, and M. K. Dougherty, Multi-instrument analysis of electron populations in Saturn's magnetosphere, J. Geophys. Res., 113, A07208, 2008.

Wu, C.S., and L. C. Lee, A theory of terrestrial kilometric radiation, Astrophys. J., 230, 621-626, 1979.

Ye, S.-Y., D. A. Gurnett, G. Fischer, B. Cecconi, J. D. Menietti, W. S. Kurth, Z. Wang, G. B. Hospodarsky, P. Zarka, and A. Lecacheux, Source locations of narrowband radio emissions detected at Saturn, J. Geophys. Res., 114, A06219, 2009.

Zarka, P., B. P. Ryabov, V.B. Ryabov, R. Prange, M. Abada-Simon, T. Farges, and L. Denis, On the Origin of Jovian Decameter Radio Bursts, in Planetary Radio Emissions IV, edited by H. O. Rucker, S. J. Bauer, and A. Lecacheux, Austrian Academy of Sciences Press, Vienna, 51-63, 1997. 
